\title{
Observations of the effects of magnetic topology on the SOL characteristics of an electromagnetic coherent mode in the first experimental campaign of W7-X
}

\author{
$\underline{\text { S. C. Liu }}^{\prime 2}$, Y. Liang ${ }^{\prime}$, P. Drews', A. Krämer-Flecken', X. Han' ${ }^{\prime 2}$, D. Nicolai', G. Satheeswaran', N. C. Wang , J. Q.

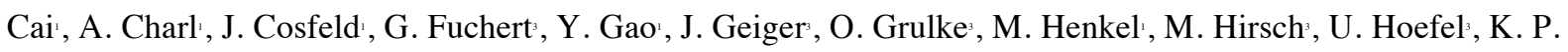 \\ Hollfeld', D. Höschen', C. Killer', A. Knieps', R. König', O. Neubauer', E. Pasch', K. Rahbarnia', M. Rack', N. \\ Sandri', S. Sereda', B. Schweer ${ }^{1}$, E. H. Wang', Y. L. Wei', G. Weir ${ }^{3}$, T. Windisch ${ }^{3}$ and W7-X Team ${ }^{*}$
}

Forschungszentrum Jülich GmbH, Institut für Energie- und Klimaforschung - Plasmaphysik, Partner of the Trilateral Euregio Cluster (TEC), 52425 Jülich, Germany

'Institute of Plasma Physics, Chinese Academy of Sciences, Hefei 230031, People's Republic of China

Max-Planck-Institute for Plasma Physics, 17491 Greifswald, Germany

Email: sh.liu@fz-juelich.de

\begin{abstract}
Turbulence is considered to play an important role in the edge cross field heat and particle transport in fusion devices. Scrape-off layer (SOL) turbulence characteristics were measured by the combined probe mounted on the multi-purpose manipulator during the first experimental campaign of W7-X. An electromagnetic coherent mode (EMCM) at $7 \mathrm{kHz}$ has been observed by multiple diagnostics in both the plasma core and the SOL and exhibits a strong dependence of the magnetic topology. As demonstrated by the measurements of the combined probe, the EMCM starts to appear at a radius of $R=6.15 \mathrm{~m}$ along the path of probe measurement and this location is shifted inwards in higher iota configurations. It propagates along the direction of electron diamagnetic drift in the far SOL with a poloidal velocity about $0.6 \mathrm{~km} / \mathrm{s}$ while it turns to the opposite direction gradually in the near SOL in the laboratory frame, but keeps a velocity of about $0.6-0.7 \mathrm{~km} / \mathrm{s}$ along the direction of electron diamagnetic drift in the plasma frame. This mode can be induced by raising the ECRH heating power in similar discharge conditions, which is probably linked to the gradient of electron temperature and pressure. The EMCM is enhanced significantly in the edge magnetic island with long connection length where the EMCM can grow up due to the long particle confinement time.
\end{abstract}

\section{Introduction}

Turbulence contributes largely to the edge cross field heat and particle transport in magnetized plasmas of fusion devices, because the transport is dominated by plasma fluctuations. The anomalous transport driven by multi-scale turbulence has been studied in both experiment and theory $[1,2]$. An electrostatic coherent mode was found to produce significant outflow of particle and heat and in consequence greatly facilitates the long H-mode sustainment in the EAST tokamak [3]. Large quasi-coherent fluctuations at $\sim 6 \mathrm{kHz}$ observed in a region with a strong density gradient and low magnetic shear drives fluctuation-induced transport which dominates the particle balance in the H-1 Heliac device [4]. Electrostatic turbulence has been studied in stellarators, such as the basic turbulence characteristics in LHD and TJ-II [5, 6], and the geodesic acoustic mode (GAM) [7, 8]. Alfvén eigenmodes (AEs) which could cause a significant loss of alpha particles from the confinement region and damage the first wall, are well investigated in TJ-II and LHD [9, 10]. Additionally, the instability driven by the ion (electron) temperature gradient is named as ITG (ETG), which is able to lead to significant ion (electron) transport [11, 12]. In TJ-II Heliac, a localized electromagnetic mode has been observed at the edge of the confinement region, which links to the presence of rational magnetic surfaces in the vicinity of the steep density gradient region [13]. Some transient magnetohydrodynamical 
(MHD) mode activities were investigated in Wendelstein-7 AS, and high cross-correlation between the MHD modes can be found in the 'bad' confinement discharges [14]. In stellarators, the edge turbulence behaviour largely depends on the 3D magnetic topology, especially magnetic islands and stochastization of the magnetic field. In the large helical device (LHD), two bifurcations of magnetic topology and transport are observed. The magnetic topology bifurcates between a nested and a stochastic magnetic field, of which the former one is characterized by the bi-directional propagation of the heat pulse with slow speed while the later one has fast heat pulse propagation and flat electron temperature. The other bifurcation is the transport in the magnetic island with high thermal diffusivity and smaller thermal diffusivity $[15,16]$. The enhanced Hurst exponent and heat flux were located at a smaller radial position than the rational surface, indicating the dependence of turbulence on the magnetic topology in TJ-II stellarator [17, 18]. The large-scale and smallscale edge turbulence in Wendelstein 7-AS were studied in detail, exhibiting an intense relationship with plasma confinement conditions [19-21].
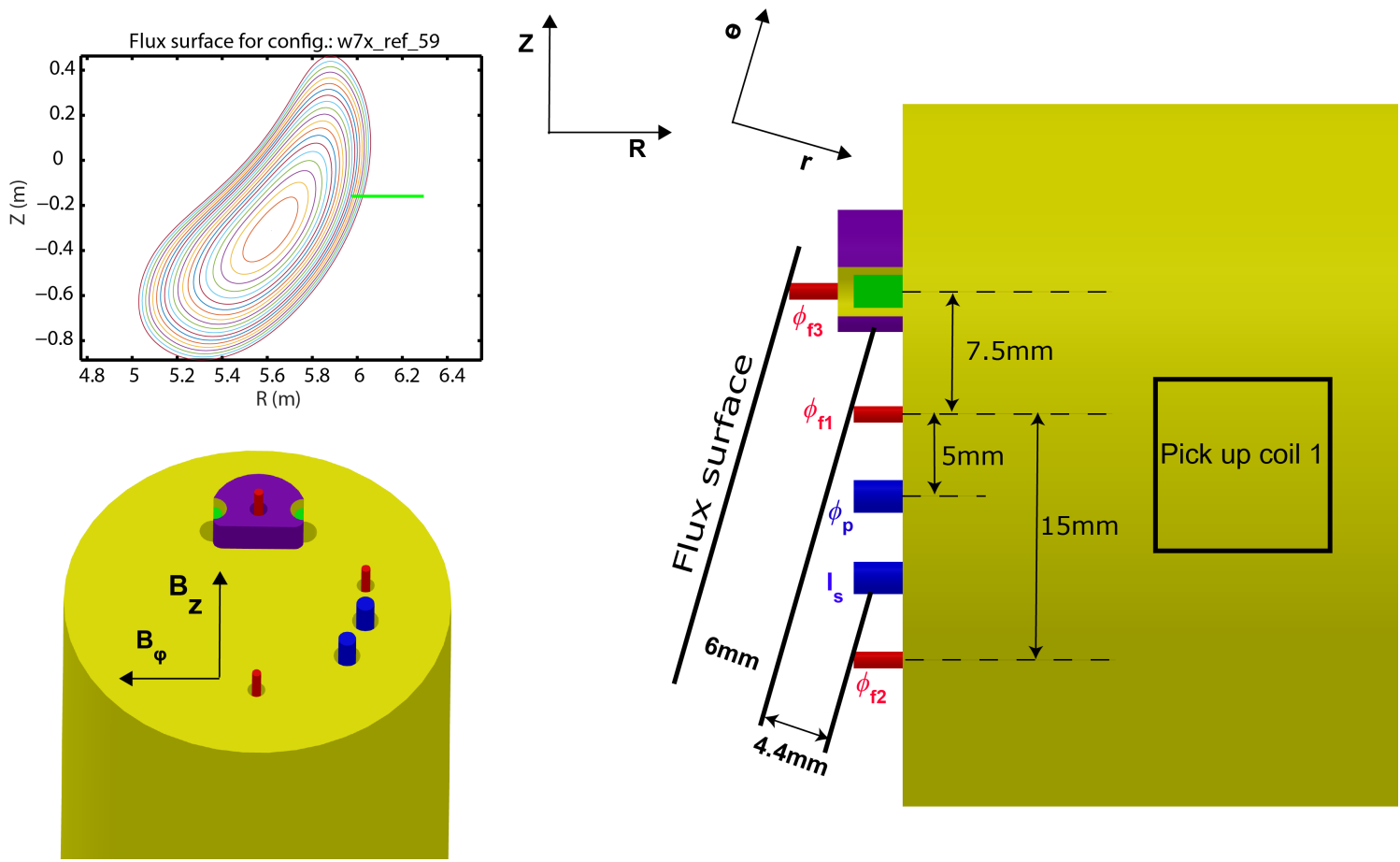

Figure 1. The sketch of the combined probe head used in OP1.1 on W7-X. The magnetic equilibrium shown in left top is standard limiter configuration, and the probe path is labelled in green line.

Wendelstein 7-X (W7-X) has large stochastic field regions and islands on the plasma edge. During the first operational phase (OP1.1) on W7-X, the scrape-off layer (SOL) plasma profiles and turbulence behaviors are measured by a combined probe mounted on a multipurpose manipulator. In this paper, the diagnostic setup is presented in Sec. 2. The characteristics of an electromagnetic coherent mode will be presented in Sec. 3. Discussion and conclusion are given in Sec. 4.

\section{Diagnostics setup}

$\mathrm{W} 7-\mathrm{X}$, whose major radius is $5.5 \mathrm{~m} \mathrm{[22],} \mathrm{represents} \mathrm{to}$ a new generation of optimized stellarators to accommodate a variety of $3 \mathrm{D}$ magnetic configurations, aiming to achieve quasi-steady state operation with plasma parameters relevant to the future fusion power plant. In order to study the edge plasma properties, a combined probe head consisting of Langmuir probes, a Mach probe and two tri-axial pick-up coils was mounted on the multi-purpose manipulator in OP1.1. The combined probe head is able to measure the electron density $n_{s}$, electron temperature $T_{s}$, plasma floating potential $\varphi$, radial electric field and parallel flow velocity, the variation of magnetic fields in directions of $(R, \phi, Z)$ and turbulence structures [23$26]$. The setup and the profile measurements of the combined probe were presented in an earlier paper[26]. Two weeks of experiments on W7-X yielded no 
significant damage on both the probe's graphite cover and the tungsten Langmuir pins. This allows an easy comparison of all discharges as the size and length of the pins were not changed. The multi-purpose manipulator is located under the outer midplane of module four at AEK40 flange with a maximum fast plunge length of $35 \mathrm{~cm}$, maximum acceleration of 30 $\mathrm{m} / \mathrm{s}^{2}$ and a maximum speed of $2.5 \mathrm{~m} / \mathrm{s}$.

As shown by the green line in the Poincaré plot in figure 1 , the combined probe is $0.167 \mathrm{~m}$ below the outer midplane. The two floating potential pins of ' $\varphi_{n}$ ' and ' $\varphi_{p}$ ' separated by $5.2 \mathrm{~mm}$ poloidally are used to calculate the poloidal structure of the turbulence. The radial correlation is derived from ' $\varphi_{i}$ ' and ' $\varphi_{s}$ ' which are separated by $6 \mathrm{~mm}$ radially. The first tri-axial pickup coil is illustrated as the black solid square in the right panel of figure 1 , which is $1.75 \mathrm{~cm}$ separated from the Langmuir pins, while the second coil is $5.12 \mathrm{~cm}$ away from the Langmuir pins. These coils are able to measure the magnetic fluctuations up to $200 \mathrm{kHz}$. The temporal resolution of the combined probe is $1 \mathrm{MHz}$.

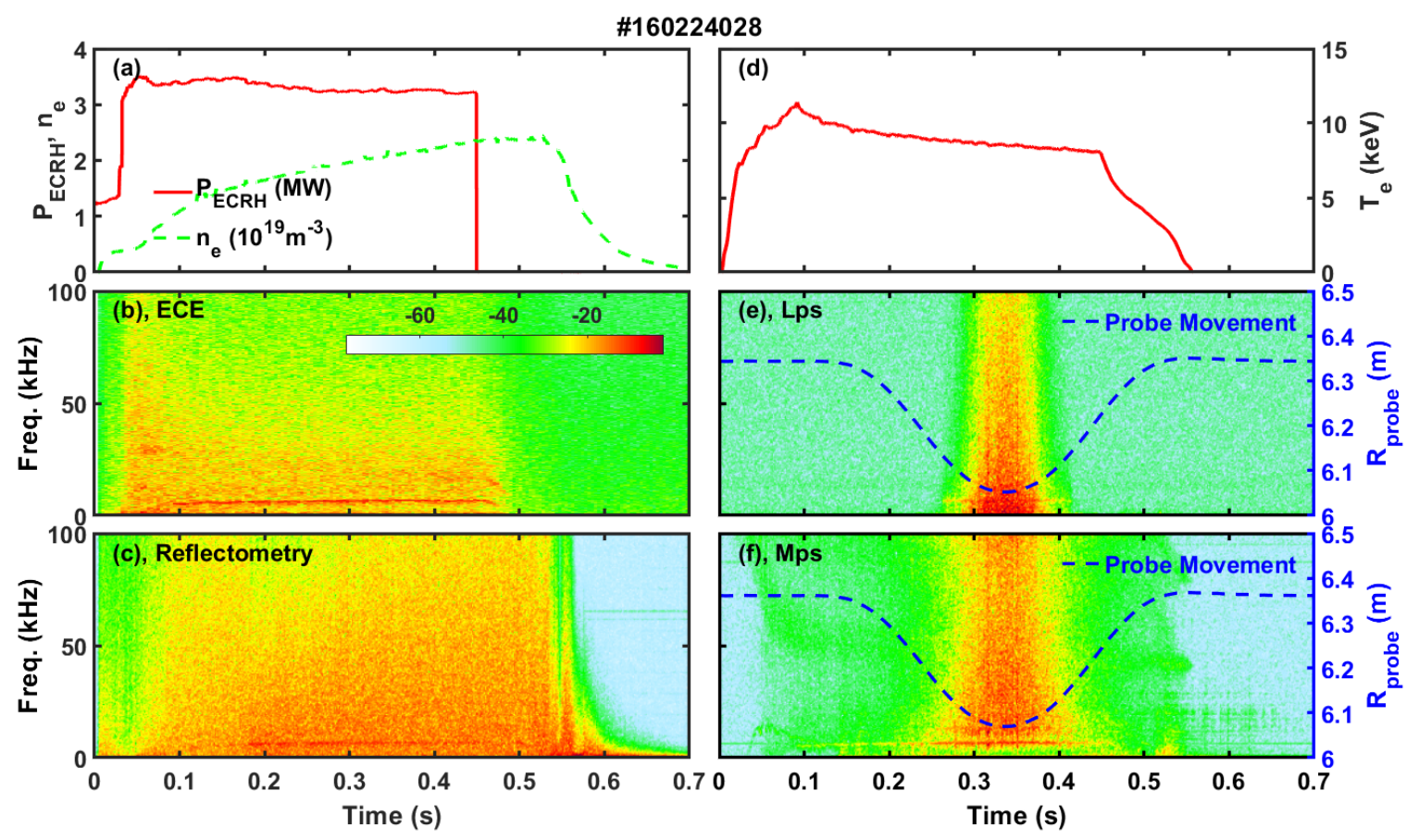

Figure 2. The plasma conditions of a discharge in OP1.1 on W7-X. (a) ECRH heating power and line averaged density; (b) power spectrum of core ECE signal; (c) power spectrum of reflectometry signal; (d) electron temperature in plasma core (channel 14) measured by ECE; auto-correlation power spectrum of Langmuir probes (e) and magnetic coil (f); The blue dashed lines in (e) and (f) are the probe position $R_{p}$. The colorbar is the logarithm to base 10 of the auto-correlation power density. Lps is the abbreviation of Langmguir probes, and Mps is the abbreviation of magnetic coils.

\section{Characteristics of an electromagnetic coherent mode}

The typical SOL turbulence characteristics have been measured by this combined probe head. As calculated by the two-point technique [27], the poloidal and radial correlation lengths of the SOL turbulence are about 5$10 \mathrm{~mm}$ and 4-6 $\mathrm{mm}$, respectively. The autocorrelation time of the SOL turbulence is around 12-15 $\mu \mathrm{s}$. Here the characteristics of a low frequency mode will be introduced.

3.1. Observation of an electromagnetic coherent mode

Figure 2 shows a discharge with electron cyclotron resonance heating $(\mathrm{ECRH})$ power of $\sim 3.2 \mathrm{MW}$ and a line averaged electron density of about $\bar{n}_{e} \sim 2 \times 10^{19} \mathrm{~m}^{3}$ during the probe plunge phase. Note that the discharge \#160224028 is the standard limiter configuration of OP1.1, and the last closed flux surface (LCFS) is 
located at $R_{\text {Lers }}=6.03 \mathrm{~m}[28,29]$. The combined probe was plunged into the plasma during 0.1-0.57 $\mathrm{s}$ with a maximum velocity of $2.5 \mathrm{~m} / \mathrm{s}$, and the probe position is illustrated as the blue dashed line in figure 2 (e) and (f) with innermost position at $R=6.052 \mathrm{~m}$, i.e., about $2 \mathrm{~cm}$ outside the LCFS. The electron temperature $T$, measured by the electron cyclotron emission (ECE), is about $9 \mathrm{keV}$ in the core plasma (channel 14). As illustrated in auto-correlation power spectrums (ACPS) of the signals from the magnetic coil on the manipulator, ECE and reflectometry, a low frequency mode near $7 \mathrm{kHz}$ can be observed obviously after the plasma is built up, i.e., when $0.1<t<0.47 \mathrm{~s}[30,31]$. The fluctuation level of this mode is almost constant in the ECE signals, while in the magnetic coil signals it increases when the probe approaching the LCFS. Figure 2 (e) displays the spectrogram of the plasma floating potential, demonstrating that the low frequency mode can only be detected in some radial position at the outer board side (OBS).

The typical fluctuation levels of the SOL floating potential $\varphi_{n}$ measured by the combined probe are shown in Figure 3. The normalized fluctuation level of the floating potential $\left|\phi_{f 1} / T_{e}\right|$ is below 1.5 in most SOL region, where $T_{e}$ is the electron temperature measured by probe. A cross-correlation has been made between the floating potential of the combined probe and the poloidal correlation reflectometry, as displayed in Figure 4. When the frequency of the reflectometry increases from $22 \mathrm{GHz}$ to $40 \mathrm{GHz}$, the corresponding density range is from $0.6 \times 10^{19} \mathrm{~m}^{-3}$ to $2 \times 10^{19} \mathrm{~m}^{-3}$ [30]. For the shot 160310039 , the central line averaged density is about $1.5-2.1 \times 10^{19} \mathrm{~m}^{-3}$ during $t=$ $0.15-0.45 s$, indicating that the reflectometry measurement probably covers a broad radial region of the core plasma. Figure 4 (b) exhibits a relatively high cross-correlation power density at the frequency of the EMCM between the core reflectometry and the SOL floating potential. The radial structure of the low frequency mode has been identified by the combined probe. As shown in Figure 5 (a), the ACPS of plasma floating potential exhibits a highlighted fluctuations at $7 \mathrm{kHz}$ when $R<6.14 \mathrm{~m}$, and the appearance of this mode is at $R=6.15 \mathrm{~m}$, about $12 \mathrm{~cm}$ outside the LCFS. The background turbulence starts to increase at $R=$ $6.09 \mathrm{~m}$, and is further enhanced at $R=6.07 \mathrm{~m}$. The cross-correlation power spectrum (CCPS) of the two floating potential pins ' $\varphi_{i}$ ' and ' $\varphi_{r}$ ' also has a similar radial turbulence structure as the ACPS. In addition, the dominant fluctuations are located in the low frequency range of $f<60 \mathrm{kHz}$, as illustrated in the ACPS and CCPS of the Langmuir probes, i.e., Figure 5 (a) and (c). Since the magnetic coils are sensitive to all magnetic fluctuations nearby, a clear mode structure can be seen in their spectrums along the whole radial path of probe. According to its features mentioned here, this low frequency mode is named electromagnetic coherent mode (EMCM).

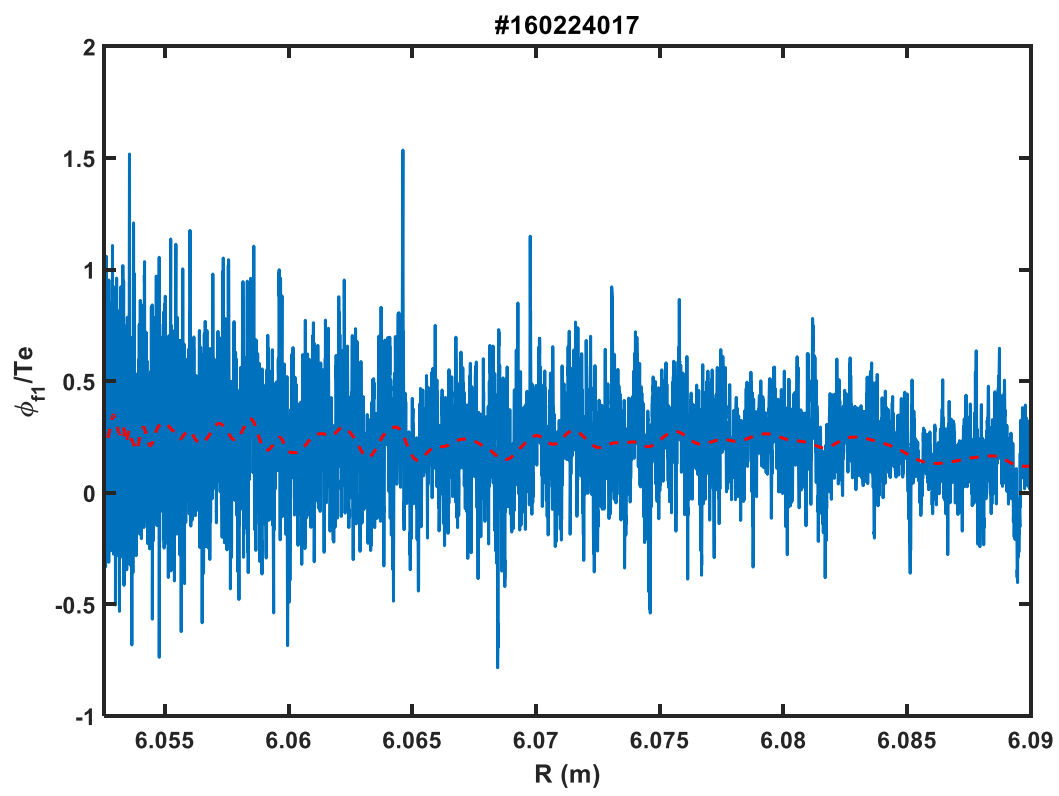

Figure 3. The typical fluctuations of floating potential for discharge \#160224017. The red dashed line denotes the mean value of $\phi_{f 1} / T_{e}$. 


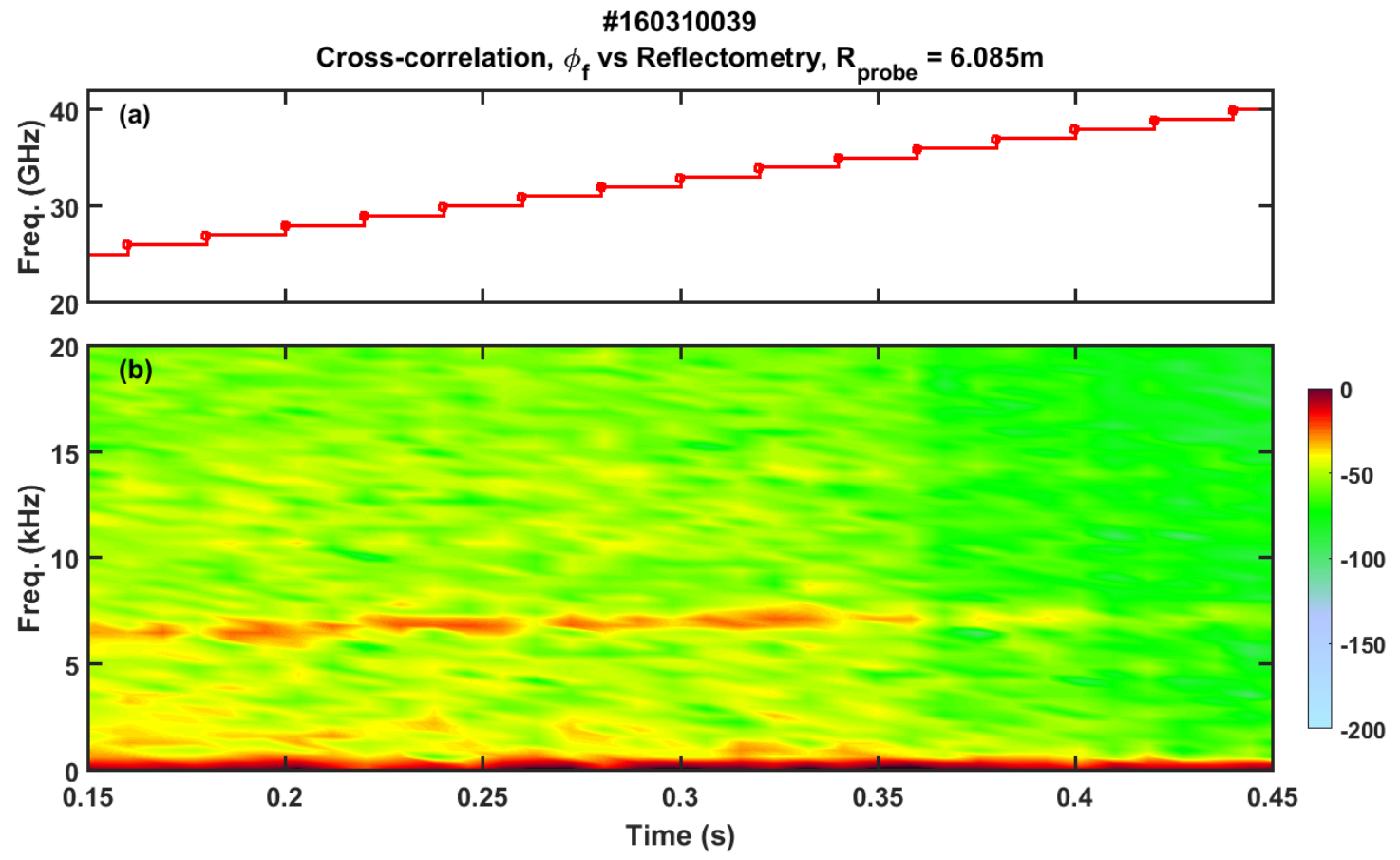

Figure 4. The cross-correlation between the floating potential of combined probe and the poloidal correlation reflectometry. (a) Frequency of the reflectometry; (b) Cross-correlation power spectrum. The position of the combined probe is fixed at $R=6.085 \mathrm{~m}$.

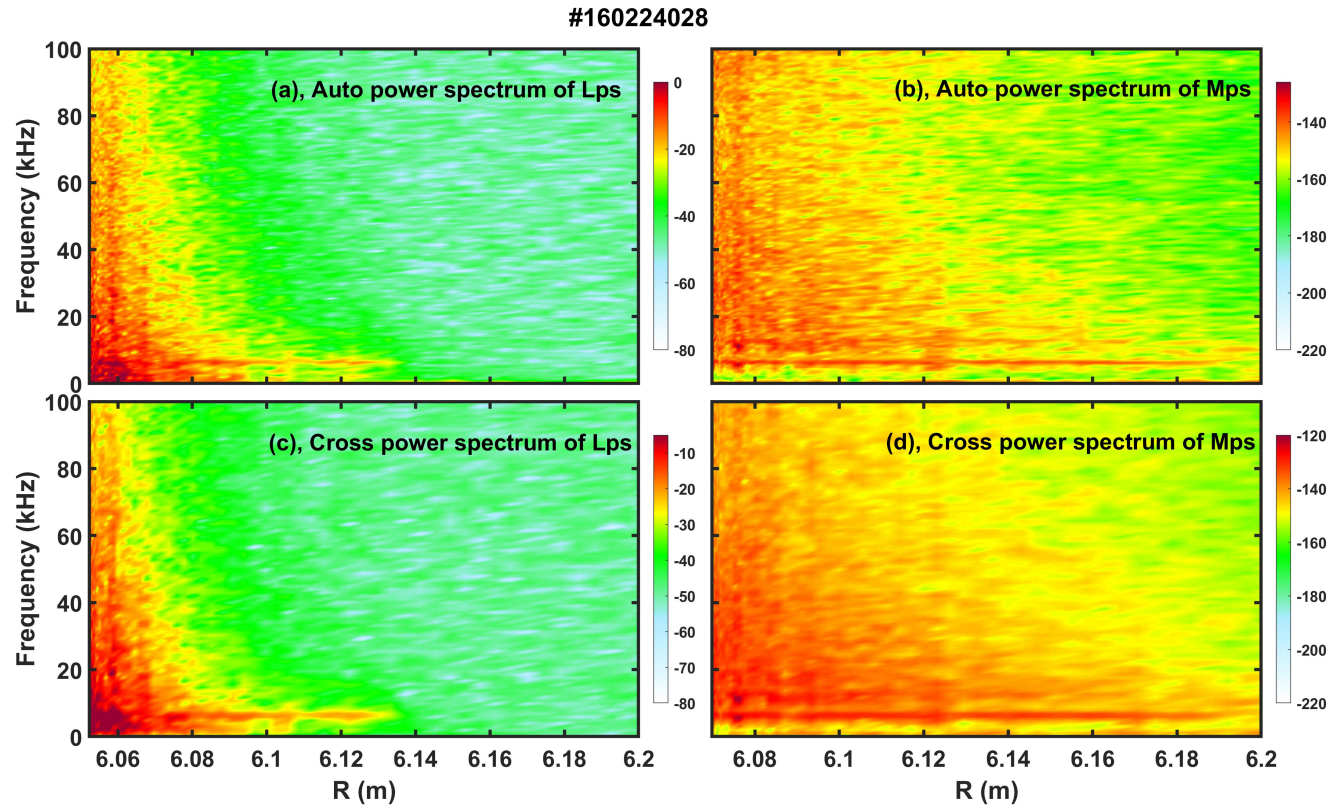

Figure 5. The auto-correlation power spectra from the plasma floating potential (a) and the magnetic coil (b) measurements of the combined probe; the cross-correlation power spectrum between two floating potential pins (' $\varphi_{n}$ ' and ' $\varphi_{\imath}$ ') (c) and two radially separated magnetic field signals along vertical direction (d). The colorbar is the logarithm to base 10 of the correlation power density $S(f)$. 


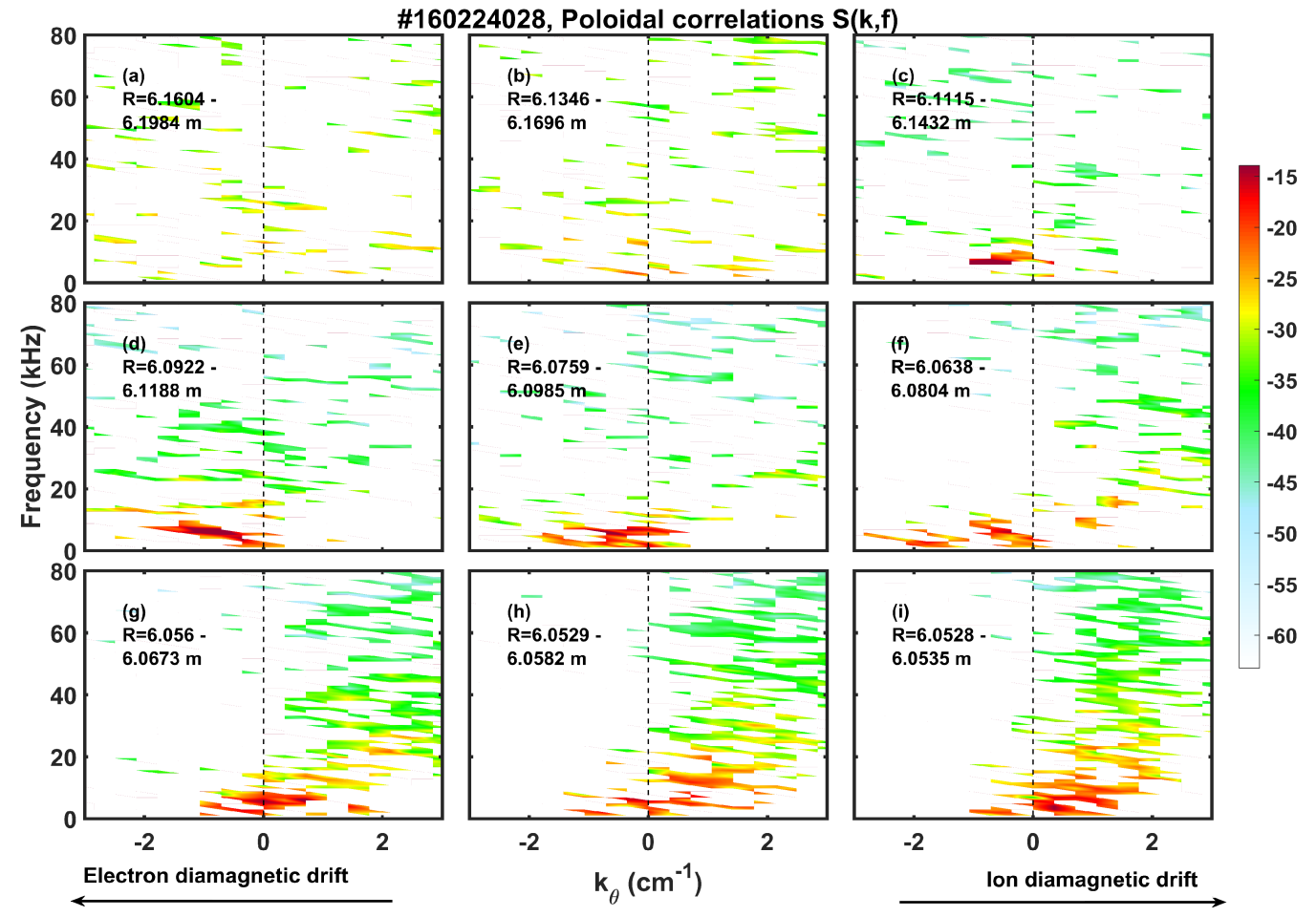

Figure 6. Poloidal correlation spectrum $S\left(k_{\theta}, f\right)$ for 9 radial positions of \#160224028. The colorbar is the logarithm to base 10 of $S\left(k_{\theta}, f\right)$. Positive $k_{\bullet}$ denotes the direction of ion diamagnetic drift, and the negative $k_{\bullet}$ denotes the direction of electron diamagnetic drift.

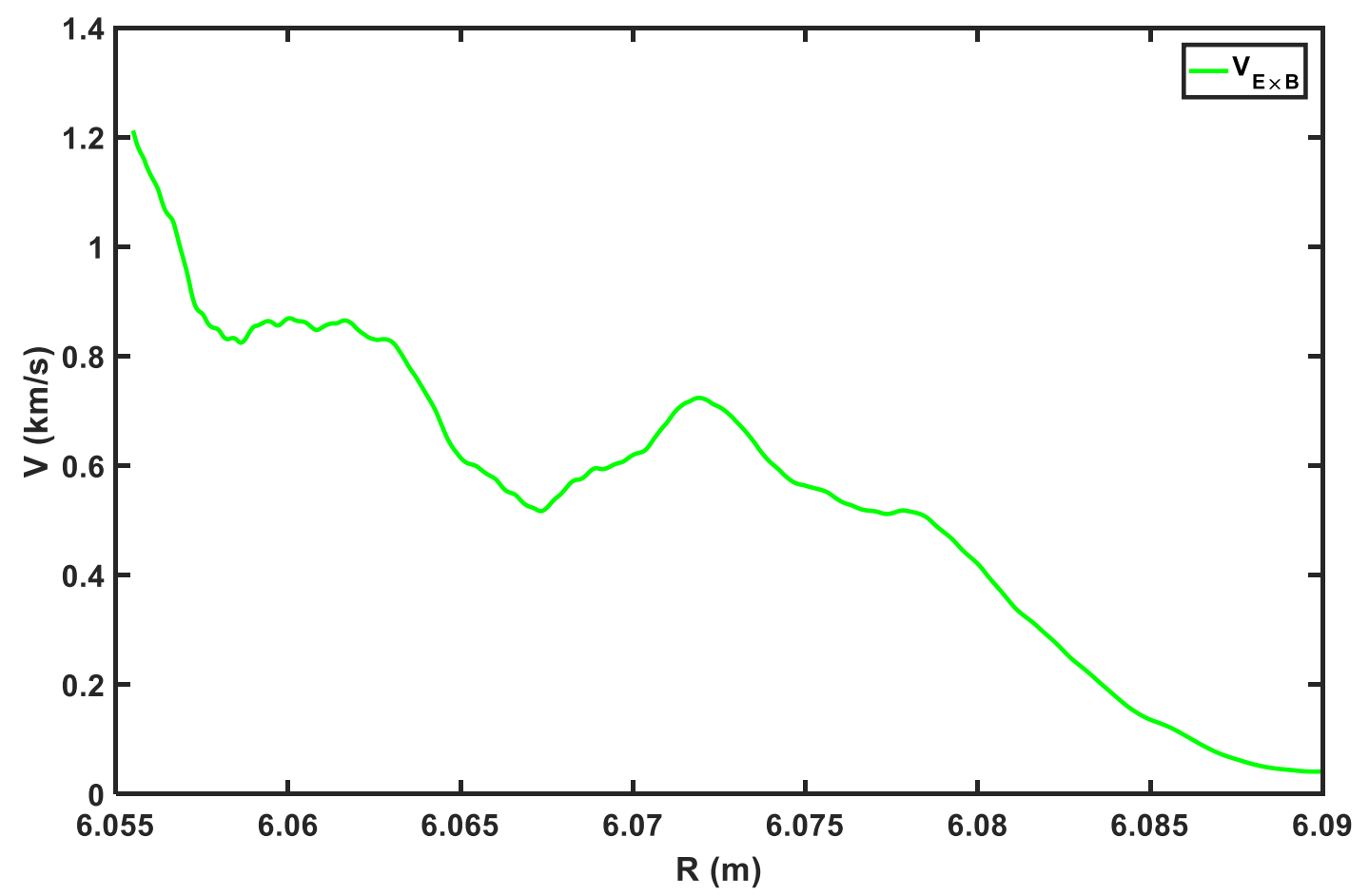

Figure 7. The poloidal electric drift velocity measured by the combined probe for shot \#160224016. 


\subsection{Propagation of EMCM}

Using the two poloidally separated floating potential pins ' $\varphi_{s}$ ' and ' $\varphi_{r}$ ', the wavenumber-frequency spectral density $S\left(k_{\theta}, f\right)$ has been computed by the two-point correlation techniques $[27,32]$. The floating potential fluctuations used in this analysis are filtered by a bandpass filter from 1 to $100 \mathrm{kHz}$, which covers the frequency range of interest. $S\left(k_{\bullet}, f\right)$ represents the power of fluctuations as a function of $f$ and $k_{\ominus}$ in the direction of two probe pins. The spectral density $S$ in Figure 6 (a) and (b) is very small and no turbulence structure can be observed in (a), because almost all of the plasma is blocked by the first wall components. In Figure 6 (c), a highlighted spectral density arises at a frequency of 7 $\mathrm{kHz}$, and poloidal wave number $k_{\bullet}$ is centred at $-0.7 \mathrm{~cm}$ in the direction of electron diamagnetic $\operatorname{drift}\left(v_{*, e}\right)$, with a phase velocity about $V_{p h}=\omega / k \approx 0.6 \mathrm{~km} / \mathrm{s}$ in laboratory frame. The location of EMCM in the space of $\left(k_{\theta}, f\right)$ is extremely explicit in the radial range of $R=$ 6.1- $6.14 \mathrm{~m}$, i.e., EMCM propagates along the direction of $v_{*, e}$. In this region the two poloidally separated floating potential pins have a high cross-correlation coefficient $\gamma$ around the frequency of $7 \mathrm{kHz}$, but relatively low $\gamma$ in the near SOL, which is similar as that of the standard limiter configuration in Figure 14. With decreasing $R-R_{L C F S}$, the spectral density of EMCM is not as highlighted as before because fluctuations of other frequencies are enhanced. Meanwhile, the poloidal wave number of the EMCM decreases and gradually moves to the direction of ion diamagnetic direction $\left(v_{*, i}\right)$. In Figure 6 (i), $R=6.053$ $\mathrm{m}$ (about $2.3 \mathrm{~cm}$ outside the LCFS), the turbulence group velocity $d \omega / d k$ is along the direction of $v_{*, i}$, roughly $1.2-2 \mathrm{~km} / \mathrm{s}$ in the laboratory frame. From the spectrum of $S\left(k_{\theta}, f\right)$, it is confirmed that the dominant frequency of fluctuations is below $60 \mathrm{kHz}$, which could contribute to the turbulent transport. The radial electric field is derived from shot \#160224016 which has the same plasma conditions as shot \#160224028 except that the pins ' $\varphi_{r}$ ' and ' $I$ ' ' are operated in the doubleprobe mode. Therefore, the poloidal electric drift is obtained by $V_{E \times B}=\overrightarrow{E_{r}} \times \vec{B} / B^{2}$, where $E_{r}=-d\left(\phi_{f}+\right.$ $\left.2.8 T_{e}\right) / d r$, as shown in Figure 7. In the far SOL region, the $V_{E \times B}$ is around $0.1 \mathrm{~km} / \mathrm{s}$ along the direction of $v_{*, i}$, and the corresponding propagating velocity of the EMCM is about $0.7 \mathrm{~km} / \mathrm{s}$ along the direction of $v_{*, e}$ in the plasma frame. In the near SOL, $V_{E \times B}$ increases up to $1.2 \mathrm{~km} / \mathrm{s}$ in the $v_{*, i}$ direction, and the velocity of the EMCM in the plasma frame is about $0.6 \mathrm{~km} / \mathrm{s}$ along the $v_{*, e}$ direction. Although the velocity of the EMCM changes from the $v_{*, e}$ direction in the far SOL to the $v_{*, i}$ direction in the near SOL in the laboratory frame, it keeps in the $v_{*, e}$ direction for both regions in the plasma frame.

During OP1.1 four Mirnov coils located in a triangular shaped plasma cross-section have been put into operation. Clear EMCM structure also can be seen in the spectrogram of the Mirnov coil signals. Besides, a fitting of the phase differences between the four coils placed poloidally suggests the primary poloidal mode number near $7 \mathrm{kHz}$ is $m=5$, propagating in the direction of $v_{*, e}$ at an angular velocity $\omega \sim-8 \mathrm{krad} / \mathrm{s}$, and the secondary mode number could be $\mathrm{m}=-8$, propagating along the direction of $v_{*, i}$ with $\omega \sim 5 \mathrm{krad} / \mathrm{s}[31,33]$. As measured by the combined probe, the propagation of EMCM in plasma frame is always in the direction of $v_{*, e}$, which is consistent with the primary poloidal mode number from Mirnov coils.

\subsection{Heating power dependence of the EMCM}

A dedicated experiment is performed to investigate the influence of heating power on the EMCM. Four discharges with different $P_{\varepsilon с к H}=2.1,2.9,3.5$ and 4.1 MW are shown in Figure 8, and the corresponding MDSplus shot numbers are 160223032, 160223033, 160223034 and 160223035 . The lowest $P_{\text {вскн }}$ shot has the smallest line averaged density $\bar{n}_{e}$ which is $0.4 \times 10^{19} \mathrm{~m}^{3}$ lower than the other shots. The increase of heating power raises the electron temperature and its radial gradient significantly in $\varrho=r_{\text {ef }} / r_{\text {Lers }}=0.15-0.32$, however in the region of $\varrho<0.07$ electron temperature has no obvious difference, as shown in Figure 8 (e). The emissions of $\mathrm{H}_{a}$ and HeI indicate similar plasma wall conditions for these discharges. Although these four shots have the same setting of coil currents of standard limiter configuration, the radial position of ECE channels could change a bit due to its dependence on the plasma pressure and the electron velocity, as shown in Figure 8 (e). Note that the electron temperature in the edge of OBS may be affected by the second harmonic of O-mode emission and the downshift of relativistic effects $[34,35]$. This could lead to some uncertainty of the radial effective radius 
of ECE channels. The combined probe is fixed at the same radial position ( $\sim 3.5 \mathrm{~cm}$ outside the LCFS) during the entire discharge of all the four shots.

The ACPSs of their floating potentials at $R=6.066$ $m$ in Figure 9 clarify the dependence of EMCM on the heating power. For the discharge with a heating power of $P_{\text {вскн }}=2.1 \mathrm{MW}$, there seems to be no fluctuation mode in the frequency range from 0 to $20 \mathrm{kHz}$. When $P_{\text {вски }}$ increases to $2.9 \mathrm{MW}$, no clear mode is observed, but enhanced background fluctuations are found even though it has a higher line averaged density than shot \#160223032. Obvious fluctuations near $7 \mathrm{kHz}$ appear in the discharge with $P_{\text {ЕсRH }}=3.5 \mathrm{MW}$, and becomes more pronounced when $P_{\text {вскн }}=4.1 \mathrm{MW}$. Since the background fluctuations at low frequencies have also high levels at $R=6.066 \mathrm{~m}$, a broadband spectrum near $0-5 \mathrm{kHz}$ is noticeable compared to the EMCM. However, the cross-correlation coefficient $\gamma$ between two floating potentials in this frequency range $(0-5 \mathrm{kHz})$ is relatively low. In contrast, $\gamma$ at $7 \mathrm{kHz}$ can be up to 0.9 , which indicates the EMCM is a strong coherent mode structure. Figure 10 shows the cross-correlation between floating potential and magnetic coil signal around $t=0.26 \mathrm{~s}$. The peaks of CCPS and $\gamma$ at $7 \mathrm{kHz}$ start to come out and grow up sharply when $P_{\text {вскн }}=$ $3.3 \mathrm{MW}$. The maximum of $\gamma$ is above 0.6 in the two higher heating power discharges. The latter three discharges have similar line averaged densities $\bar{n}_{e}$, indicating that the EMCM can be induced by high heating power.

As mentioned in Figure 8, the radial gradient of the electron temperature at OBS increases about 50\% when $P_{\text {вснн }}$ changes from 2.1 MW to 4.1 MW. However no obvious variation of $\bar{n}_{e}$ has been found when $P_{\text {вскH }}$ changes from 2.9 MW to $3.5 \mathrm{MW}$, but the EMCM becomes remarkable. It should be pointed out that the ACPSs of ECE signals express similar results as Langmuir probes, i.e., the onset of the EMCM is observed only when the heating power is at $P_{\text {вскн }}=3.5$ and 4.1 MW. Furthermore, EMCM is strong in the ECE channels of 9-14, but for other channels it is difficult to detect this mode due to very weak fluctuations. In Figure 8 (e), the large gradient of $T$ is located in the region of channel 9-13 ( $\varrho=0.11-0.32)$, indicating that the EMCM may be linked to the gradient of electron temperature or pressure.

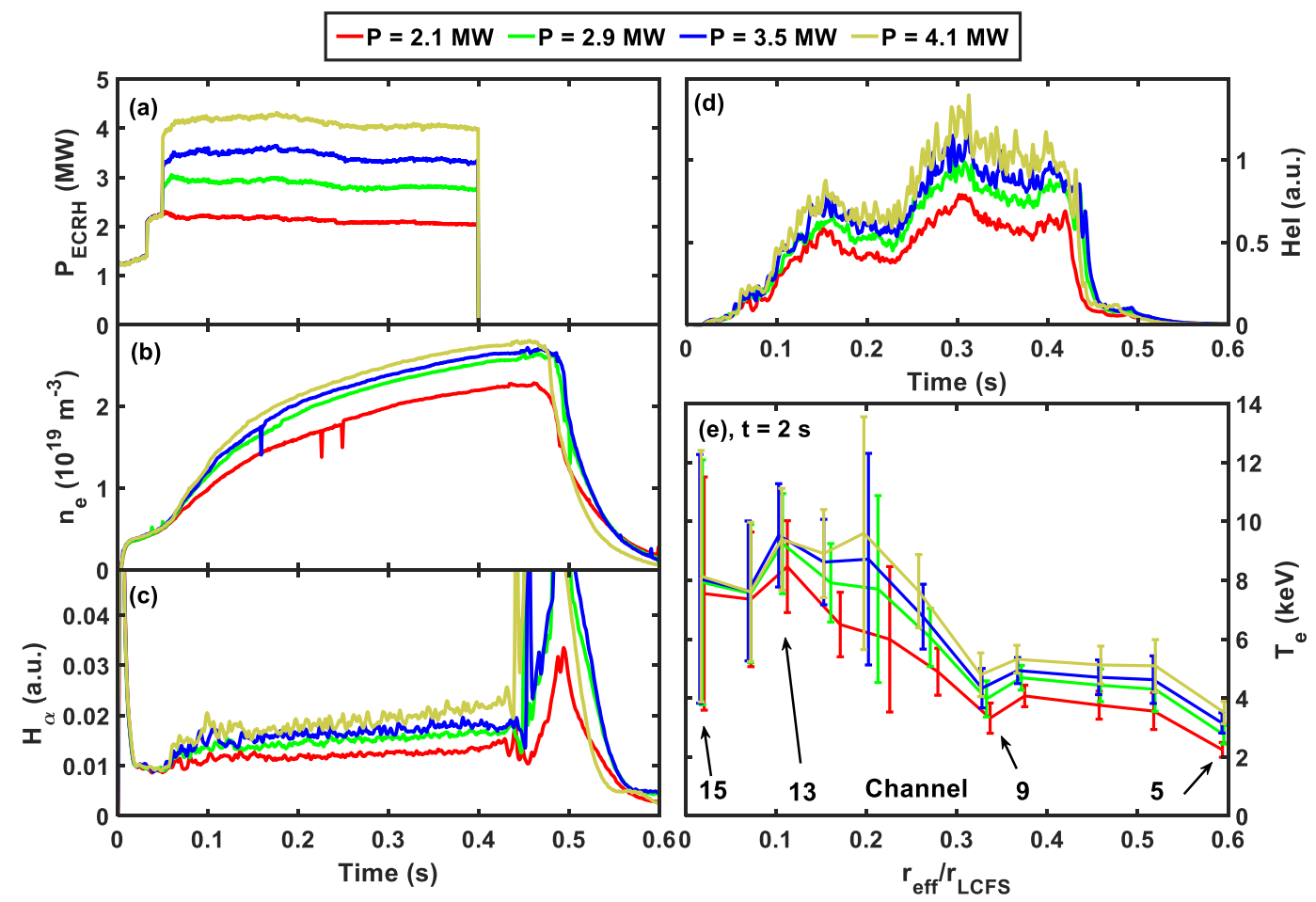

Figure 8. Plasma discharge conditions. (a) ECRH heating power; (b) line averaged density; (c) $\mathrm{H}_{\alpha}$ emission; (d) HeI line emission; (e) radial profile of $T$, given by ECE at $t=0.2 \mathrm{~s}$, and the solid lines are the guide lines for $T$, variation. 


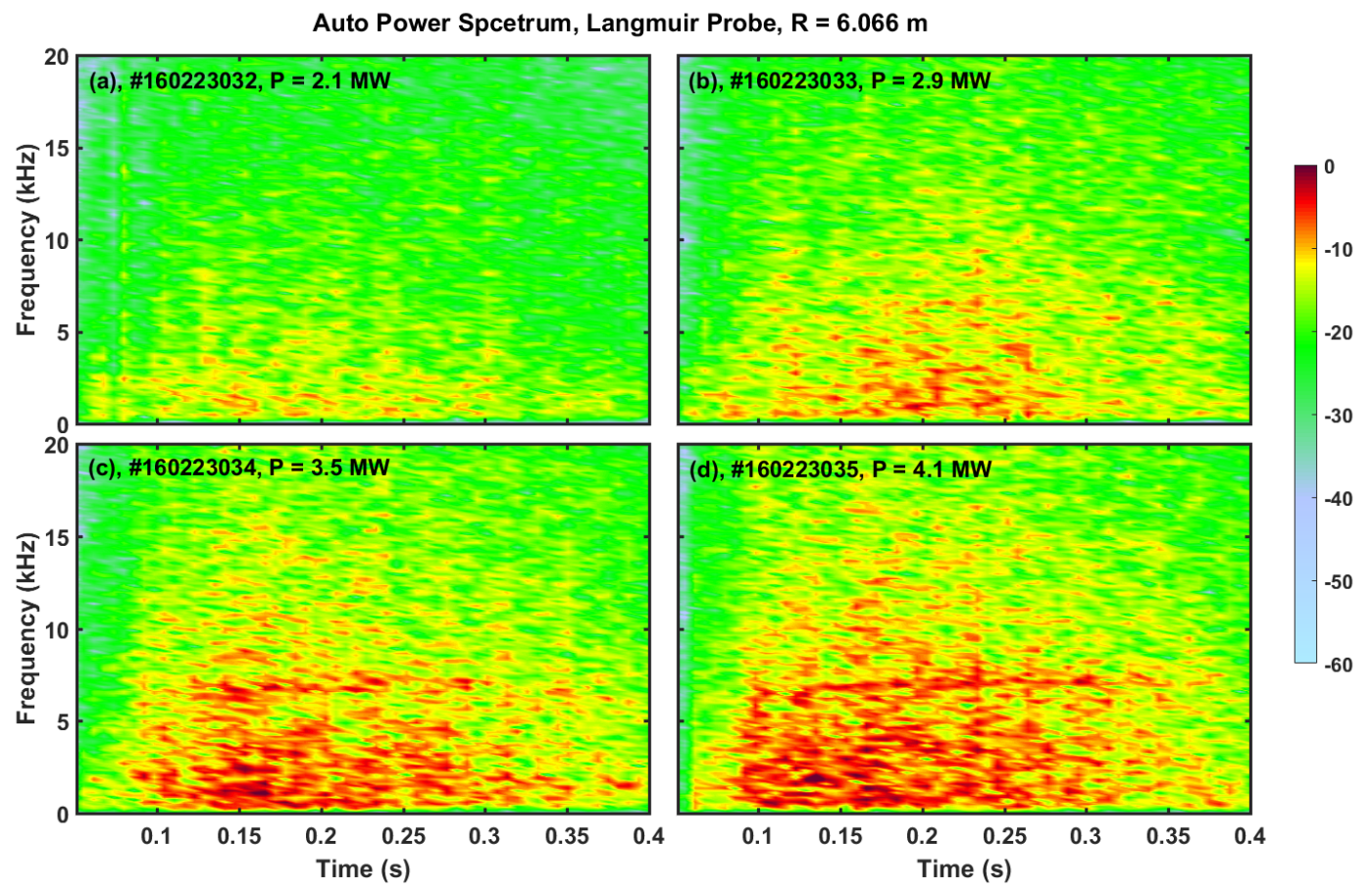

Figure 9. The auto-correlation power spectra of floating potential for an ECRH heating power scan experiment, with the combined probe staying at $R=6.066 \mathrm{~m}$. The colorbar is the logarithm to base 10 of the auto-correlation power density $S(f)$.

$--P=2.1 M W--P=2.8 M W-P=3.4 M W-P=4 M W$
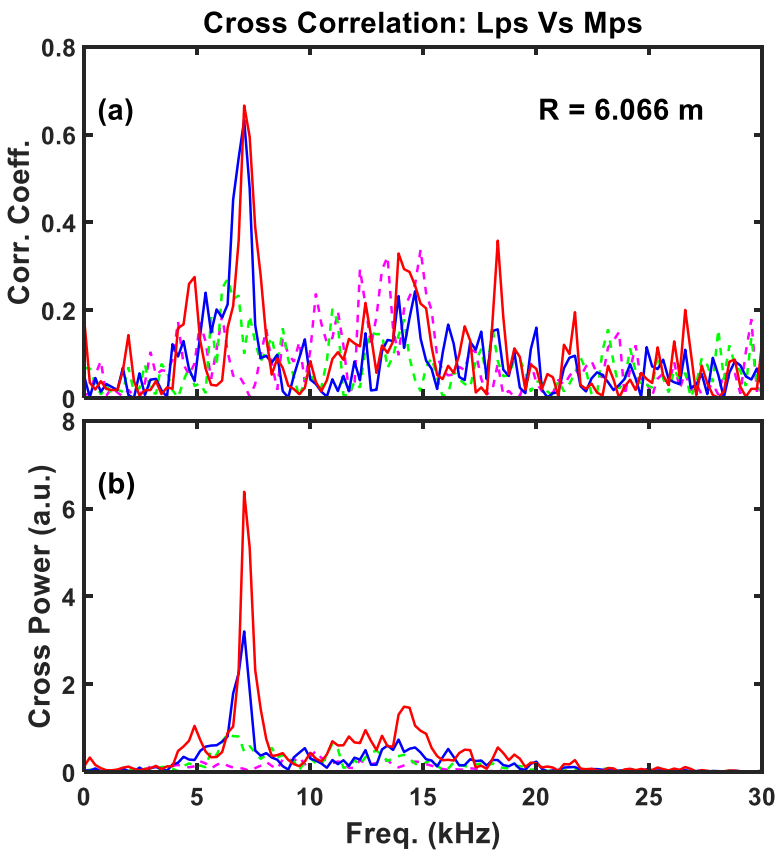

Figure 10. The cross-correlation between floating potential and toroidal magnetic field signal. (a) cross-correlation coefficient; (b) cross-correlation power. Lps is the abbreviation of Langmguir probes, and Mps is the abbreviation of magnetic coils. 
3.4. Dependence of the EMCM on magnetic topology

In OP1.1 the magnetic configuration has been changed from low iota to higher iota step by step to study its effects on the plasma performance. The magnetic configuration is controlled by the planar coils and nonplanar coils on W7-X [28,36]. Discharge conditions of configuration scan experiments are shown in Figure 11, with ECRH heating power of $2 \mathrm{MW}$ and $\bar{n}_{e}=2.3-$ $2.7 \times 10^{19} \mathrm{~m}^{3}$. The line averaged density $\bar{n}_{e}$ increases slightly with shot number. Figure 11 (c) and (d) give the electron temperature and density profiles measured by Thomson scattering (TS). A flat $n$, profile is observed when $\varrho<0.6$, while the profile of $T$, exhibits a steep gradient from $\varrho=0.1-0.8$. The electron density and temperature profiles measured by the Langmuir probe are shown in Figure 12. According to the tripleprobe theory, electron temperature is calculated by $T_{e}=\left(\phi_{p}-\phi_{f}\right) / \ln 2$ and electron density is obtained from $n_{e}=I_{s} /\left(0.49 e A_{e f f} \sqrt{T_{e} / m_{i}}\right)$, where $A_{e f f}$ is the effective collected area of the probe pin, $\phi_{p}, \phi_{f}$ and $I_{s}$ are illustrated in Figure 1 [37]. As calculated from the field line tracer code, the major radii of the LCFS along the path of combined probe for the standard limiter, iota- 8 and iota-12 configurations are $6.03,6.01$ and 6.0 $\mathrm{m}$, respectively, which can be seen from the inward shift of $T$, and $n_{c}$ in higher iota configurations. Because both the heating power and line averaged density of these discharges are low for W7-X, the electron density $\left(\sim 0.6 \times 10^{18} \mathrm{~m}^{3}\right)$ and temperature $(15-25 \mathrm{eV})$ in the SOL are in the same level when compared to that measured in the TEXTOR tokamak [32]. Due to the fixed space positions of TS channels, the effective radius of each channel will become larger, which leads to the outward shift of the profiles of TS, as illustrated in Figure 11. Figure 13 shows the rotational transform profiles of the three magnetic configurations, with the $5 / 6$ (here 5 is the toroidal mode number and 6 is the poloidal mode number) island chain inside the LCFS and the 5/5 island chain outside the LCFS. In higher iota configurations, the iota profiles are shifted inward significantly and the iota values also increase.
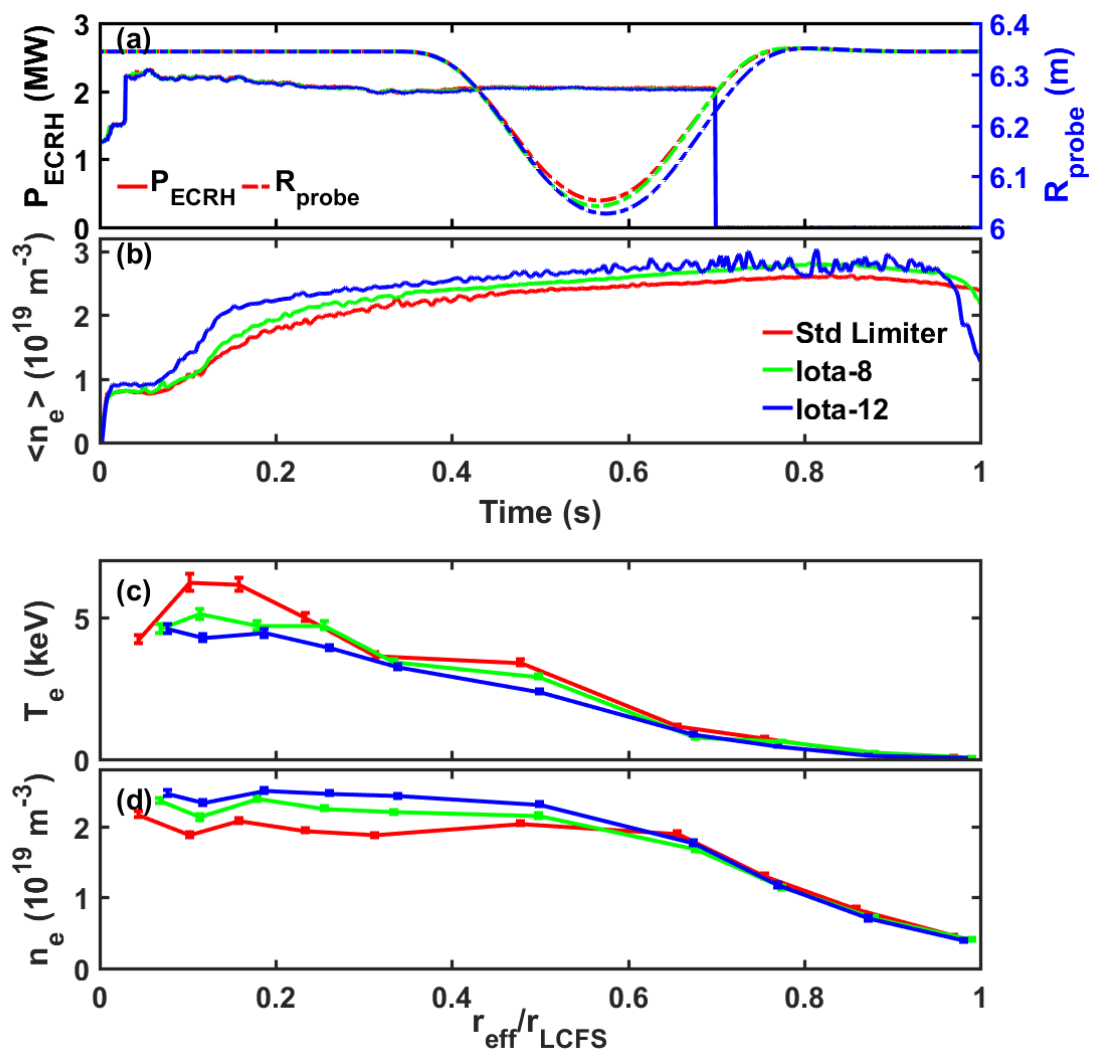

Figure 11. Discharge conditions for configuration scan experiment. (a) ECRH heating power; (b) line averaged density; (c) and (d) are electron temperature and electron density measured by Thomson scattering, respectively, with the solid line denoting the guide lines of the parameters variation. The probe plunge is indicated as the dashed lines in (a). 

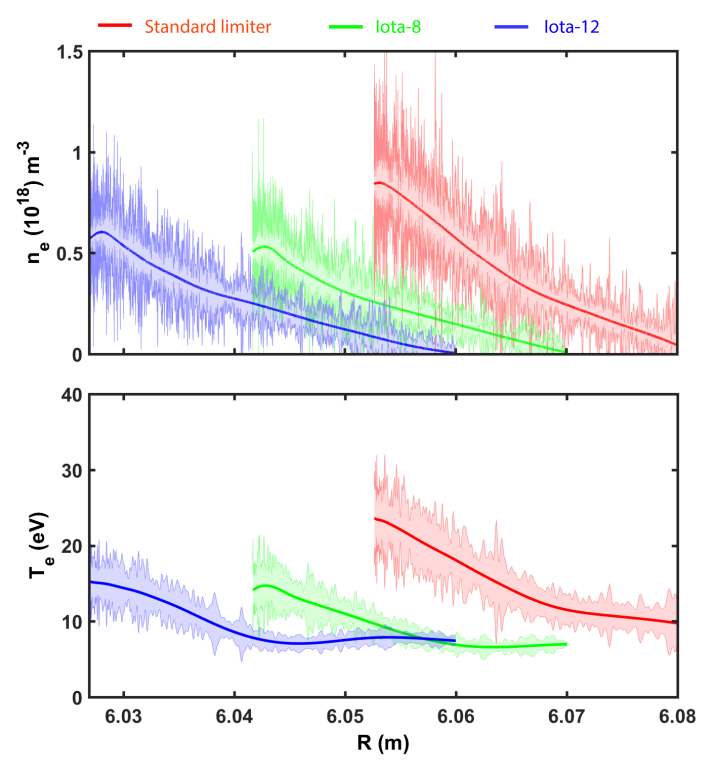

Figure 12. SOL profiles of $n$ and $T$. measured by the combined probe. The shaded area denotes the standard deviation of parameters.

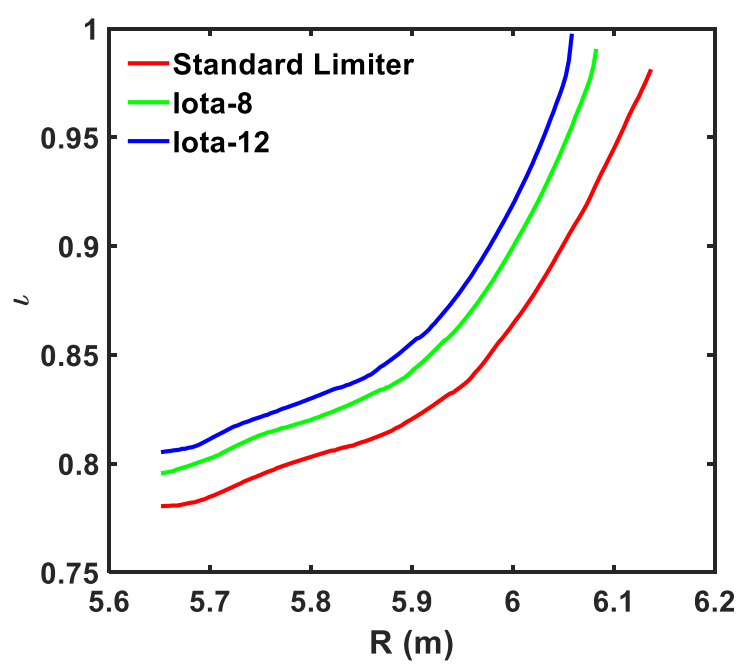

Figure 13. Iota profiles of the three magnetic configurations along the path of fast probe calculated by the field line tracer.

The cross-correlation between the floating potential and the magnetic field signals of the combined probe has been analyzed to study the effect of magnetic topology on the EMCM, as shown in Figure 14. The floating potential is a localized measurement while the magnetic coil signal is nonlocalized. As a result, the cross-correlation power spectral density (CPSD) could be large, but the cross-correlation coefficient $\gamma$ is low in some radial regions at the frequency of EMCM, because the high CPSD only appears on the magnetic signal. In the standard limiter configuration, a $7 \mathrm{kHz}$ mode starts to emerge at $R \sim 6.15 \mathrm{~m}$, displaying a $\gamma$ large than 0.6 in the radial range of $R=6.11-6.15 \mathrm{~m}$. Meanwhile, the connection length $L$ increases rapidly to $\sim 15 \mathrm{~m}$ at the position of the onset of EMCM, then $\gamma$ increases with the connection length before it reaches $80 \mathrm{~m}$. When $L>80 \mathrm{~m}$ the field lines end on the limiters of W7-X. The maximum of $\gamma$ can be up to 0.9 . For the iota-8 configuration, high correlation is also observed in the radial region with increasing connection length, and the onset of the EMCM is at $R$ $=6.12 \mathrm{~m}$. In the highest iota configuration iota-12, a 
high $\gamma$ larger than 0.8 appears in a broad radial region of $R=6.06-6.09 \mathrm{~m}$ with an averaged connection length of $160 \mathrm{~m}$. Compared to the standard limiter and iota-8 configurations, the CPSD of EMCM is much higher in the iota-12 configuration, representing a larger fluctuation amplitude. It should be noted that the frequency of the EMCM increases with edge iota, i.e., $7 \mathrm{kHz}$ for standard limiter, $7.8 \mathrm{kHz}$ for iota- 8 and $9 \mathrm{kHz}$ for iota-12. The phase difference between floating potential and the vertical component of magnetic field is between $-0.3 \pi$ to $-0.8 \pi$ for the high correlation region.

The Poincaré plots of the three configurations are calculated by field line tracer and displayed in Figure 15 [38]. In order to reveal the complete magnetic equilibrium, this calculation is performed by considering only the torus (meshed models id: 164) of W7-X. The green dashed line denotes the radial path of the combined probe, the black dotted line is the location of the LCFS and the red line is the normalized connection length. A magnetic island is located in the path of the combined probe for all configurations. In the standard limiter configuration, the island is very narrow (1.5 cm width) and far away from the LCFS (with a distance of $14.5 \mathrm{~cm}$ ) in the radial direction. However, this island becomes much wider and closer to the LCFS in higher iota configurations, with island width $\sim 6.5 \mathrm{~cm}$ for both, and distance about 7.8 and 6.2 $\mathrm{cm}$ for iota- 8 and iota-12 configurations, respectively. The connection length inside the magnetic island is pretty long if not blocked by the first wall. During the calculation of the connection length, all the components of the first wall in OP1.1 are taken into account, as shown in the magenta solid line of Figure 14 and the red line of Figure 15. In the iota-12 configuration, the long connection length $(\sim 160 \mathrm{~m})$ is found inside the magnetic island, which leads to high cross-correlation power and coefficient at the EMCM frequency, i.e., strong fluctuations of EMCM. Figure 15 (d)-(f) shows the cross-correlation coefficient between two floating potentials near the EMCM frequency, indicating that the localized fluctuation level of EMCM is enhanced inside the magnetic island. The amplitude of fluctuation $A$ increases with confinement time $\tau$ if the growth rate of the turbulence is constant. On the other hand, the particle confinement time $\tau_{p} \approx L / C$, is directly proportional to the connection length $L$ in the SOL, where $C$ is the ion sound speed [39]. In consequence, the fluctuation amplitude $A$ will increase with connection length $L$ inside the magnetic island where the fluctuations of the EMCM are extremely large.

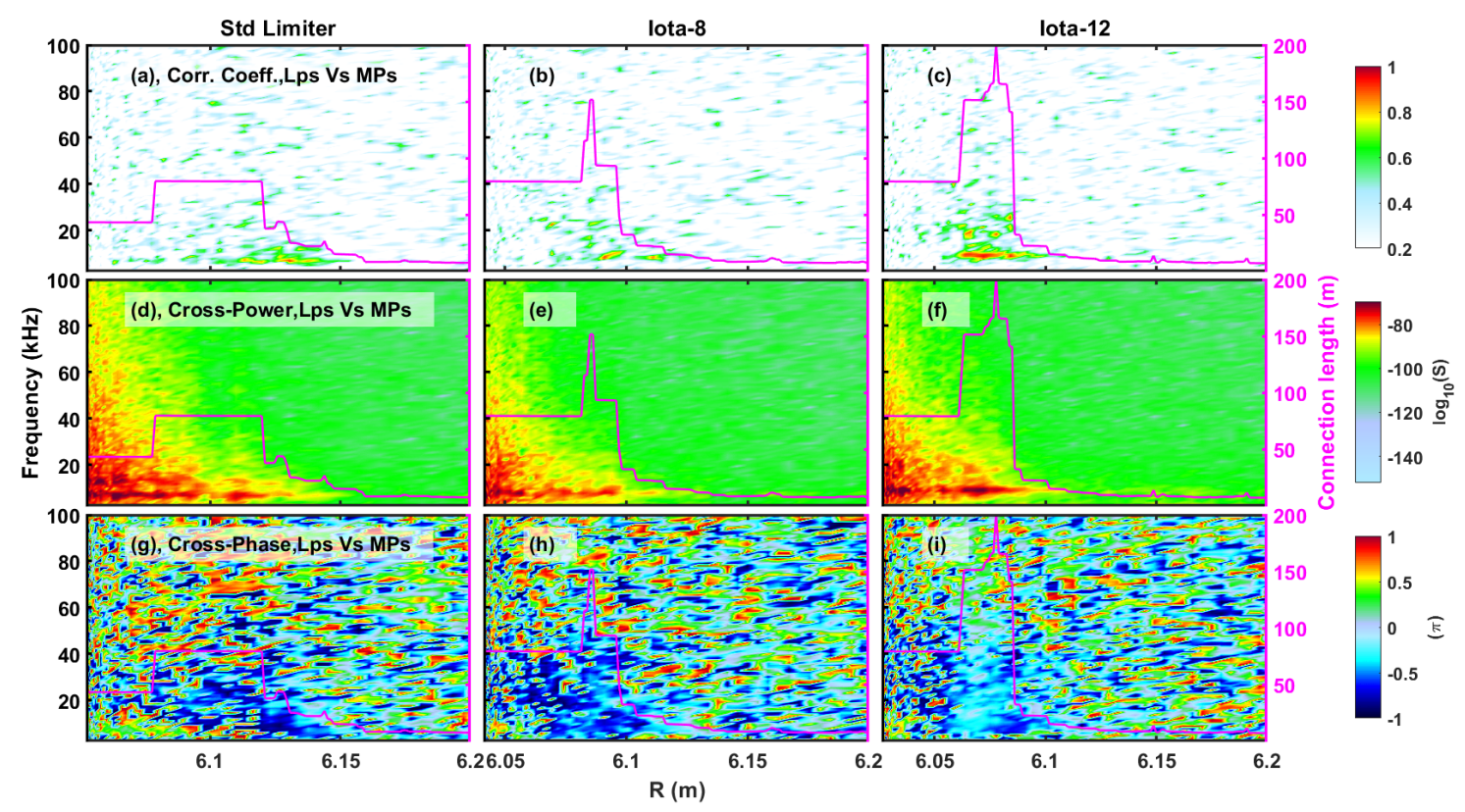

Figure 14. The cross-correlation between floating potential and magnetic coil signal. From left to right the standard limiter, iota- 8 and iota-12 configurations are shown. From top to bottom the cross-correlation coefficient, the 
logarithm of cross-correlation power and the cross-correlation phase are shown. The unit of cross-correlation phases is $\pi$.The magenta solid line represents connection length of each configuration.

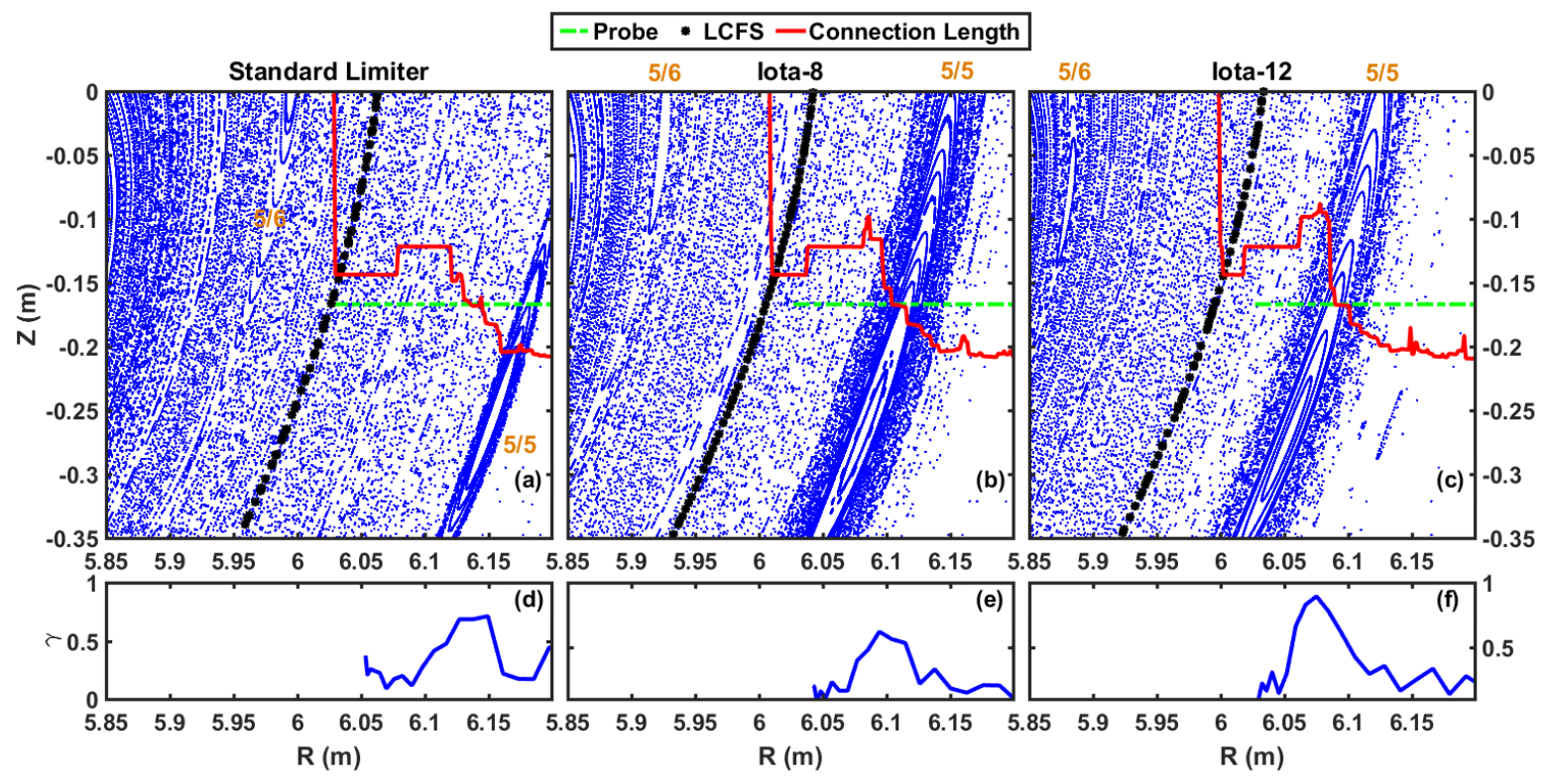

Figure 15. Poincaré plot of the magnetic equilibrium and normalized connection length for the three configurations (a)-(c). The black dashed line denotes the position of the LCFS. The cross-correlation coefficient near the EMCM frequency between two floating potentials are shown in (d)-(f).

\section{Discussion and conclusion}

The SOL structures of the EMCM strongly depend on edge topology. When configuration changes from standard limiter to higher iota, besides the inward shift of the EMCM radial position, there is also a broad radial region with the relatively high cross-correlation power density and cross-correlation coefficient, which is located inside the large magnetic island. Due to the long connection length inside the island, this mode has a longer confinement time to grow up therefore an enhanced EMCM is observed in this long connection length region. In addition, the EMCM can be induced and enhanced by high ECRH heating, which is confirmed by the measurements of Langmuir probes and magnetic coils of the combined probe in the near SOL. The electron temperature and its radial gradient measured by ECE increase significantly at OBS when raising the heating power $P_{\text {вскн. }}$. Meanwhile, in high $P_{\text {вскн }}$ cases the clear EMCM structure also can be found in the ECE channels located in the region with a sharp electron temperature gradient. The cross-correlation between the floating potential of the combined probe and the poloidal correlation reflectometry, exhibits a relatively high cross-correlation power density at the frequency of the EMCM between the core electron density and the SOL floating potential.
The SOL characteristics of an electromagnetic coherent mode have been investigated in the first experimental campaign of $\mathrm{W} 7-\mathrm{X}$. The dominant frequency of fluctuations in the SOL is below $60 \mathrm{kHz}$, as measured by the Langmuir probes and the magnetic coils on manipulator. The EMCM starts to appear around $R=6.15 \mathrm{~m}$ in standard limiter configuration and this location is shifted inward in the higher iota configurations. In standard limiter configuration, the cross-correlation between two floating potential pins, which are separated poloidally, demonstrates that this EMCM propagates along the direction of electron diamagnetic drift in the far SOL region with a phase velocity about $0.6 \mathrm{~km} / \mathrm{s}$ in the laboratory frame. When the probe plunges deeper, the phase velocity of EMCM decreases gradually and finally changes to the opposite direction in the near SOL. However, in the plasma frame the EMCM always propagates along the electron diamagnetic drift direction at a velocity around 0.6-0.7 $\mathrm{km} / \mathrm{s}$. These observations are consistent with the poloidal mode number and its rotation direction calculated from the Mirnov coils.

Currently it is difficult to expound the mechanism of the EMCM due to the diagnostic limitations in OP1.1. Instead, we would like to report the experimental observations of this mode here and attract 
more attention to study it in the following experiments and simulations. A brief summary of this mode is as below: (1) The EMCM can be observed by both Langmuir probes and magnetic coils, indicating its electromagnetic property. (2) The EMCM can be induced by raising the ECRH heating power, which may relate to the increase of profile gradient, i.e., the enhancement of the free energy source. (3) This mode appears in both the SOL region and the plasma core, and has strong dependence on the magnetic topology in the SOL. (4) The EMCM propagates along the direction of electron diamagnetic drift in the plasma frame in the SOL region.

The particle transport driven by this mode is not clear yet due to the limitation of the combined probe head used in OP1.1. A new combined probe head, consisting of optimized Langmuir probe pins, Mach probe, magnetic coil, differential coil and ion sensitive probes, has been developed for OP1.2a of W7-X to study the edge turbulence structure and related transport. Using the new probe head which is able to plunge into the LCFS, the most interesting characteristics of the EMCMs including mode propagation and the induced particle transport can be measured, which will shed more light on the interpretation of EMCM and its impact on W7-X.

\section{Acknowledgement}

This work has been carried out within the framework of the EUROfusion Consortium, has received funding from the Euratom research and training programme 2014-2018 under grant agreement No 633053, and was supported by the National Natural Science Foundation of China under Grant Nos. 11405213 and 11605235. The views and opinions expressed herein do not necessarily reflect those of the European Commission.

\section{Reference}

[1] Gusakov E. Z. et al 2013 Plasma Phys. Control. Fusion. 55124034

[2] Connor J. W. et al 1994 Plasma Phys. Control. Fusion. 36719

[3] Wang H. Q. et al 2014 Phys. Rev. Lett. 112 185004

[4] Michael C. A. et al 2017 Plasma Phys. Control. Fusion. 59024001

[5] Dewhurst J. M. et al 2008 Plasma Phys. Control. Fusion. 50095013

[6] Hidalgo C. et al 2002 New J. Phys. 451
[7] Ido T. et al 2010 Plasma Phys. Control. Fusion. 52 124025

[8] Castejon F. et al 2016 Plasma Phys. Control.

Fusion. 58094001

[9] Jimenez-Gomez R. et al 2011 Nucl. Fusion 51 033001

[10] Toi K. et al 2009 Plasma Sci. Technol. 11377

[11] Jenko F. et al 2000 Phys. Plasmas 71904

[12] Dorland W. et al 2000 Phys. Rev. Lett. 855579

[13] Jimenez J. A. et al 2006 Plasma Phys. Control.

Fusion. 48515

[14] Pokol G. et al 2007 Plasma Phys. Control.

Fusion. 491391

[15] Ida K. et al 2015 Nat Commun 65816

[16] Ida K. et al 2016 Nucl. Fusion 56092001

[17] van Milligen B. P. et al 2017 Nucl. Fusion 57 056028

[18] van Milligen B. P. et al 2016 Nucl. Fusion 56

016013

[19] Basse N. P. et al 2005 Phys. Plasmas 12012507

[20] Basse N. P. et al 2003 Nucl. Fusion 4340

[21] Grulke O. et al 2001 Phys. Plasmas 85171

[22] Pedersen T. S. et al 2016 Nat Commun 713493

[23] Liang Y. et al 2017 Nucl. Fusion 57066049

[24] Nicolai D. et al 2017 Fusion Eng. Des. ISSN 09203796

[25] Satheeswaran G. et al 2017 Fusion Eng. Des. ISSN 09203796

[26] Drews P. et al 2017 Nucl. Fusion 57126020

[27] Beall J. M. et al 1982 J. Appl. Phys. 533933

[28] Geiger J. et al 2015 Plasma Phys. Control.

Fusion. 57014004

[29] Pedersen T. S. et al 2015 Nucl. Fusion 55

126001

[30] Krämer-Flecken A. et al 2017 Nucl. Fusion 57 066023

[31] Windisch T. et al 2017 Plasma Phys. Control. Fusion. 59105002

[32] Xu Y. et al 2007 Nucl. Fusion 471696

[33] Rahbarnia K. et al in 43rd EPS Conference on

Plasma PhysicsLeuben, Belgium, 2016), p. P4.011.

[34] Weir G. M. et al in 43rd EPS Conference on

Plasma PhysicsLeuben, Belgium, 2016), p. P4.009.

[35] Sato M. et al 1995 Jpn J Appl Phys 234 L708

[36] Otte M. et al 2016 Plasma Phys. Control. Fusion. 58064003

[37] Hutchinson I. H. 2002 Principles of plasma diagnostics (Cambridge ; New York: Cambridge University Press)

[38] Bozhenkov S. A. et al 2013 Fusion Eng. Des. 88

2997

[39] Stangeby P. C. 2000 The plasma boundary of magnetic fusion devices (Bristol: Institute of Physics Publishing) 